\title{
Das Verhältnis einiger Salzlösungen gegen eine alkalische Lösung des Antimontrioxyds.
}

\section{Von}

M. C. Harding.

Setzt man eine alkalische Lösung des Antimontrioxyds zu verschiedenen Salzlösungen, so erhält man Niederschläge, deren Natur ich untersucht habe. Eine Lösung ron Antimontrioxyd in einer verdünnten Lösung des Kaliumbydroxyds wurde angewandt.

Wird eine verdünnte Lösung des Wasserstoffplatinchlorids zu einer im Uberschufs vorhandenen alkalischen Lösung des Antimontrioxyds gesetzt, so erhält man eine braune Flüssigkeit, die bald einen schwarzbraunen Niederschlag ausscheidet. Dieser wurde abfiltriert, ausgesüfst, getrocknet und darauf auf folgende Weise qualitativ analysiert.

Durch Zersetzung mit verdünnter Salzsäure erhält man einen Rest von metallischem Platin, während eine ebenso grofse Menge desselben Metalls in Lösung als Platinoxydsalz übergeht. Ein Präparat, welches im ganzen 35.38\% Pt enthielt, gab bei dieser Behandlung $17.70 \% \mathrm{Pt}$. Die salzsaure Lösung enthält das Antimon als Trioxyd. Übersättigt man nämlich diese Lösung mit Kaliumhydroxyd, so wird bei Zusatz von Silbernitrat der für Antimontrioxyd eigentümliche schwarze Niederschlag gewonnen. Das Präparat enthält also Platin als Platinoxydul und Antimon als Trioxyd.

Vollständig ibereinstimmende Resultate ergab die quantitative Analyse von einer Reihe von Präparaten wohl nicht; in den meisten Fällen aber lag die relative Menge von Platin und Antimon in der Nähe des Verbältnisses 1 Atom Platin auf 2 Atome Antimon. 
Über den schwarzen Niederschlag, welchen Goldoxydsalze mit Ïberschufs emer alkalischen Lösung des Antimontrioxyds hervorbringen, wird von mehreren Autoren ${ }^{1}$ berichtet. Unter diesen Autoren deutet nur Gmelis-KradT ${ }^{2}$ in einem kleinen Artikel mit dem Titel: „Antimonsaures Goldoxydul“" an, dafs das Präparat neben Gold auch Antimon entbält. Ich habe die Reaktion unter verschiedenen Bedingungen untersucht, und eine Reihe von Niederschlägen qualitativ und quantitativ analysiert. Als Resultate dieser Versuche führe ich an:

Ist die Goldlösung sauer oder alkalisch, so enthält der Niederschlag Goldoxydul nebst einer höchst variablen Mlenge von Antimontrioxyd (ich habe von 6.55\% $-26.00 \%$ Sb gefunden). Ist die Goldlösung mit Kaliumbydroxyd neutralisiert worden, so enthält der Niederschlag nebst dem Goldoxydul eine nicht so variable Menge Antimontrioxyds (in 4 verschiedenen Präparaten wurden 33.49, 32.51, 31.97 und $33.50 \% \mathrm{Sb}$ auf 51.33, 52.98, 53.28 und 53.70\% Au gefunden). Die relative Menge von Gold und Antimon liegt in diesen Präparaten in der Nähe des Verhältnisses: 1 Atom Gold auf 1 Atom Antimon.

Wird Überschufs der Goldlösung angewandt, so enthält der Niederschlag im wesentlichen nur Goldoxydul.

Wird eine Lösung von Quecksilberchlorid za einem Überschufs der alkalischen Antimontrioxydlösung gesetzt und der dabei hervorkommende Niederschlag erwärmt, so enthält dieser metallisches Quecksilber nebst geringen Spuren von Antimon. Dieselbe Reaktion kommt beim Stehen ohne Erwärmung hervor. Der Niederschlag enthält in diesem letzten Falle sofort eine variable Menge von Quecksilberoxydul, welches erst beim Stehen in freies Quecksilber verändert wird. - Gegen Mercurosalze verhält sich die Lösung in derselben Weise.

Wird bei der Reaktion mit Quecksilberchlorid Unterschufs der alkalischen Antimonlösung angewandt, so scheidet sich kein freies Quecksilber aus, der Niederschlag aber enthält Quecksilberoxydul.

Eine alkalische Lösung des Antimontrioxyds verhält sich also einer Lösung von Quecksilberchlorid gegenüber wie eine salzsaure

1 Rose, Handb. der anal. Chem. (6. AuA. 1867) 1, 428; Barfoed, Larebog $i$ den anal. Chem. (2. Udg. 1880) S. 254, 415.

'Gmenn-Kraut, Handb. d. anorg. Chem. (6. Aufl. 1875) 3, 1039; Dammer, Handb. d. anorg. Chem. (1893) 3, 773. 
Liösung des Zinnoxyduls, nur mit dem Unterschiede, dals die Reaktion nicht so energisch im ersten als im letzten Falle vor sich geht.

Das Verhältnis gegen Silberlösungen ist von Rose ${ }^{1}$ und besonders von Piluitz ${ }^{2}$ näher studiert worden. Piluitz fand, dals der schwarze Niederschlag ein Gemenge von Silber und Antimon enthält, vielleicht auch eine Verbindung zwischen diesen beiden Elementen.

Wird eine verdünnte wässerige Lösung von Kupfersulfat zum Überschufs der alkalischen Lösung des Antimonoxyds gesetzt, so erhält man eine blaue Lösung, von der beim kurzen Stehen ein hellgrüner Niederschlag niederfällt.

Die qualitative Analyse zeigte, dafs dieser Niederschlag aus Cupriantimonit besteht.

Die quantitative Analyse wurde in der Art ausgeführt, dals eine salzsaure Lösung des Niederschlages mit Schwefelwasserstoff gesättigt wurde. Die ausgefällten Schwefelverbindungen wurden dann durch Schwefelnatrium auf die gewöhnliche Weise getrennt. Das Schwefelantimon wurde auf einem bei $100^{\circ}$ getrockneten und gewogenen Filter bestimmt und dann - um die letzte Feuchtigkeit und den freien Schwefel zu entfernen - in einem $\mathrm{CO}_{2}-$ Strome bei $200^{\circ}$ erwärmt. Das Kupfer wurde als Cuprosulfid bestimmt, indem das Cuprisulfid mit Schwefel in einem Wasserstoffstrome erhitzt wurde.

$\begin{array}{lccc} & \text { I. } & \text { II. } & \text { Ber. für } \mathrm{CuSb}_{9} \mathrm{O}_{4} \text { : } \\ \mathrm{Cu} & 17.23 & 17.01 & 17.39 \% \\ \mathrm{Sb} & 65.88 & 65.98 & 65.72 \%\end{array}$

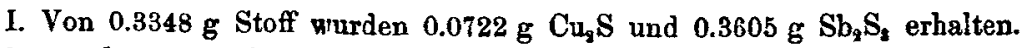
Von diesem letzten verloren $0.2878 \mathrm{~g}$ bei Erwärmung in $\mathrm{CO}_{9} 0.0413 \mathrm{~g}$.

II. Von $0.2470 \mathrm{~g}$ Stoff wurden $0.0526 \mathrm{~g} \mathrm{Cu}_{2} \mathrm{~S}$ und $0.2588 \mathrm{~g} \mathrm{Sb} \mathrm{S}_{\mathrm{g}}$ erhalten. Von diesem letzten verloren $0.1910 \mathrm{~g}$ bei Erwärmung in $\mathrm{CO}_{2} 0.0226 \mathrm{~g}$.

Diesen Analysen zufolge besteht der Niederschlag aus Cupriantimonit von der Formel: $\mathrm{CuSb}_{2} \mathrm{O}_{4}$.

Ein Präparat von derselben Formel wird von Benzelıus ${ }^{3}$ erwähnt, aber er führt keine Darstellungsweise an.

${ }^{1}$ Rose, Handb. der anal. Chem. (6. Aufl. 1867) 1, 429.

${ }^{2}$ Pillitz, Z. anal. Chem. (1882) 21, 27, 496.

8 Berzelius, Lehrb. d. Chem. (3. Aufl. 1835) 4, 581. 
Cupriantimonit kann mittels einer alkalischen Lösung von Kaliumantimonyltartrat dargestellt werden. Löst man 15 Teile von diesem Stoffe in 300 Teilen $8 \%$ iger Kaliumhydroxydlösung auf und setzt zu dieser Lösung 2 Teile krystallisiertes Kupfersulfat in verdünnter wüsseriger Lösung, so scheidet die blaue Flüssigkeit nach kurzem Stehen einen hellgrünen Niederschlag von Cupriantimonit aus.

Cupriantimonit bildet ein hellgrünes Krystallmehl. Es verliert bei mälsiger Erwärmung im Tiegel ohne Deckel zunächst etwas Antimonoxyd, welches sich verflüchtigt; danach entzündet sich der Rest, und ein Gemenge von Antimontrioxyd und Kupferoxyd bleibt zurück. Bei Erwärmung ohıe Zutritt der Luft enthält der Rest reduziertes Kupfer. Cupriantimonit ist in Salzsäure, Weinsäure und Citronensäure löslich.

Wird eine nicht zu starke Lösung von Eisenchlorid zu einer alkalischen Lösung des Antimontrioxyds gesetzt und das Ganze einen Tag hingestellt, so hat die Flüssigkeit sich meistens mehr oder weniger stark rötlich gefärbt. Eine erkennbare Menge von Eisen hat sich gelöst. Wird eine Lösung von Antimonoxyd in Kaliumhydroxyd angewandt, so ist die Fähigkeit des Eisens, in Lösung zu gehen, eine gröfsere, als wenn eine Lösung in Natriumhydroxyd benutzt wird. Es ist doch keine beständige Lösung. Mit Carbonaten des Calciums, Baryums, Strontiums, Magniums und Bleis geschüttelt, entfärbt sie sich bald. Bei Erwärmung fällt ein gelbbrauner Niederschlag nieder, der Eisen als Oxyd und Antimon als Trioxyd enthält. Das Verhältnis zwischen Eisen und Antimon in diesen Niederschlägen ist stark variabel. - Von stark rötlich gefärbten Lösungen wurden Präparate erhalten, in welchen 35-40\% Eisen und 23-28\% Antimon vorhanden waren.

Über das Verhältnis einer Lösung ron Kaliumpermanganat gegen eine alkalische Lösung von Antimontrioxyd ist von REYNoso ${ }^{1}$ berichtet worden; er fuhrt jedoch blofs an, dals eine Reduktion des Kaliumpermanganats stattfindet. Hierzu kann bemerkt werden, dafs der Niederschlag bedeutende Mengen von Antimon enthalten kann. Das Mangan ist als Peroxyd und das Antimon als Trioxyd zugegen, aber die Menge von diesen beiden Elementen ist stark

1 Reysoso, Chem. Centralbl. 1851, 528. 
variabel. Ich habe in verschiedenen Präparaten 21-31\% Mangan und 23-28\% Antimon gefunden.

Die alkalische Antimonlösung reduziert gelöste Chromate, so dafs sich Chromoxydverbindungen bilden.

Wird endlich eine Lösung von Stannochlorid zum Überschuls der alkalischen Antimonoxydlösung gesetzt, so dafs das ausgefallte Stannohydroxyd sich wieder löst, so erhält man bei Erwärmung einen grauschwarzen Niederschlag, der nebst einer geringen Menge von Zinn nur freies Antimon enthält. Bei passender Konzentration und Erwärmung setzt sich das Antimon als ein Metallspiegel auf der Seite des Glases ab. Während in den oben angeführten Reaktionen die alkalische Antimonoxydlösung oxydiert wird, ist hier das Verbältnis umgekehrt; hier wird die Antimonverbindung reduziert.

Kopenhagen, Chem. Laboratorium der Universität, Februar 1899.

Bei der Redaktion eingegangen am 26. Februar 1899. 